\title{
Reflexiones acerca de las enfermedades geriatría y el servicio de medicina interna
}

\section{Reflections about geriatric diseases and the internal medicine service}

\section{Reflexões sobre doenças geriátricas e o serviço de medicina interna}

\author{
Marlene Patrícia Yanqui-Ramos ${ }^{\mathrm{I}}$ \\ marlenepatricia0488@gmail.com \\ Jimena Vanessa Recalde-Rosado II \\ jime_realder@hotmail.com \\ Carlos Alberto Vela-Trejo ${ }^{\mathrm{III}}$ \\ drcarlosvela_647@hotmail.com \\ Luis Ángel Jimbo-Yépez ${ }^{\mathrm{IV}}$ \\ luisjy@hotmail.com
}

Recibido: 10 de enero de 2019 *Aceptado: 10 de marzo de 2019 * Publicado: 05 de abril de 2019

I Médico, Postgradista Medicina Familiar y Comunitaria, Hospital Teófilo Dávila, Machala, Ecuador.

II Médico, Residente de Medicina Interna, Hospital General de Manta, Manta, Ecuador.

III Médico, Postgradista Medicina Familiar y Comunitaria, Hospital Teófilo Dávila, Machala, Ecuador.

${ }^{\text {IV }}$ Médico, Residente de Medicina Interna, Hospital General de Manta, Manta, Ecuador. 


\title{
Resumen
}

La tercera edad es un período en que las necesidades de salud son más crecientes, por los cambios fisiológicos que aparecen en el declinar de la vida, hace que sin dudas requieran las acciones intersectoriales, para mejorar la salud, calidad de vida y supervivencia. Se realizó una revisión bibliografía relacionada con las enfermedades geriátricas y el servicio de medicina interna.

Palabras claves: Geriatría; adulto mayor; servicio de medicina interna.

\begin{abstract}
The third age is a period in which health needs are increasing, because of the physiological changes that appear in the decline of life, which undoubtedly require intersectoral actions to improve health, quality of life and survival. A literature review related to geriatric diseases and the internal medicine service was carried out.
\end{abstract}

Keywords: Geriatrics; Elderly; internal medicine service.

\section{Resumo}

A terceira idade é um período em que as necessidades de saúde estão aumentando, por causa das mudanças fisiológicas que aparecem no declínio da vida, que, sem dúvida, exigem ações intersetoriais para melhorar a saúde, a qualidade de vida e a sobrevivência. Uma revisão da literatura relacionada às doenças geriátricas e ao serviço de medicina interna foi realizada.

Palavras-chave: Geriatria; idoso serviço de medicina interna. 


\section{Introducción}

El envejecimiento se caracteriza por cambios y transformaciones producidos por la interrelación entre factores genéticos y ambientales, asociados a factores de riesgo a lo largo de la vida. La manifestación de estos cambios está dados en declinaciones en el estado de salud, condicionantes de su deterioro funcional. La adecuada interacción del adulto mayor con el medio y los vínculos extra familiares mejoran su calidad de vida y supervivencia, por ende, los aleja de los temores y preocupaciones que implica envejecer. (Louro Bernal, Bayarre Vea, \& Álvarez Lauzarique, 2015)

Esencialmente el envejecimiento constituye una victoria de la vida, en el que las personas que viven en mejores condiciones de vida pueden arribar a edades avanzadas, pero a su vez implica un desafío para diversas esferas de la sociedad como son: los servicios, la producción material, la seguridad social, entre otros. (González Rodríguez \& Cardentey García, 2015)

Aunque desde el punto de vista demográfico es el aumento en la proporción de personas de edad avanzada en relación con el resto de la población, no solo es importante hacer referencia a esta proporción, sino también a la disminución de niños y jóvenes menores de 15 años. (Rodríquez Cabrera , y otros, 2015) Con relación a este último aspecto constituye una situación problémica para el propio sistema de salud, toda vez que debe velar y garantizar la calidad de vida de las personas envejecidas, pues compromete su desempeño en las más elementales actividades de la vida cotidiana., (Rodríguez Cabrera, Collazo Ramos, Calero Ricard, \& Bayarre Vea, 2013)

La tercera edad es un período en que las necesidades de salud son más crecientes, por los cambios fisiológicos que aparecen en el declinar de la vida. La participación comunitaria para mejorar la salud del adulto mayor debe ser vista desde la intersectorialdad como proceso social que juega un papel primordial, pues la dependencia o falta de autonomía del adulto mayor en varios niveles: económico, afectivo y físico, el requerimiento de ayuda en lo referente al acceso a medicamentos, disponibilidad de alimentos, necesidad del lavado de ropa, asistencia social a domicilio, acceso a recreación, ayuda económica, la presencia de enfermedades crónicas, degenerativas e incapacitantes, las que a su vez son progresivas, de larga duración, difíciles de controlar y por lo tanto, más costosas de tratar, hace 
que sin dudas requieran de un apoyo importante de la comunidad y de las acciones intersectoriales, para mejorar la salud, calidad de vida y supervivencia. (Benítez Pérez , 2017)

La Medicina Interna, entendida como especialidad, proporciona una atención clínica completa y científica, que integra los aspectos fisiopatológicos, diagnóstica y da tratamiento con los humanos enfermos, mediante el adecuado uso de los recursos médicos disponibles. El internista debe poseer profundos conocimientos científicos, amplia experiencia clínica y demostrada capacidad de perfeccionamiento profesional y responsabilizarse en el cuidado personal y continuo de los enfermos. Actúa como consultor con otros especialistas y, a su vez, es capaz de integrar las opiniones de éstos en beneficio del cuidado integral y global del paciente. Ningún proceso morboso o problema clínico que el enfermo padezca le debe parecer ajeno a su incumbencia y responsabilidad aunque, como es lógico, logre la experiencia y actuación de otros especialistas siempre que sea preciso.

La atención al paciente enfermo hospitalizado (incluye a pacientes con enfermedades crónicas descompensadas o con estados morbosos intercurrentes), el apoyo de consultoría en atención primaria u otras especialidades y la atención al paciente crónico complejo son los procesos clave en las unidades de medicina interna. (Pacheco Mejías, 2017)

En la actualidad, en varios países del mundo, el estudio de la problemática del anciano constituye un elemento de primordial importancia y aparece como factor decisivo en las investigaciones médicas. Esto se debe al incremento internacional de la población mayor de 65 años, calculada en más de 290 millones de personas con un aumento creciente en las últimas décadas. (Reyes Tornes \& Card Huerta, 1996)

La República del Ecuador es uno de los países de América Latina que ha entrado de lleno en la transición demográfica, como respuesta a los cambios sociales y económicos que tuvieron lugar en las tres últimas décadas: un mayor desarrollo económico, la difusión de los avances médicos y el mayor acceso de la población a los servicios de salud, incluyendo el conocimiento y el uso de métodos de planificación familiar y el mayor acceso a la educación, entre otros. (Estudio sobre la protección social de la tercera edad en Ecuador, 2007)

147 Vol. 5, núm. 2, abril 2019, pp.144-159

Marlene Patricia Yanqui Ramos, Jimena Vanessa Recalde Rosado, Carlos Alberto Vela Trejo, Luis Ángel Jimbo Yépez 


\section{La geriatrización de los servicios y de los profesionales de la salud}

A lo largo de la historia, los médicos siempre atendieron enfermos ancianos, aunque en número limitado. A pesar de ello, ya se identificaban en estos pacientes maneras especiales de expresar las enfermedades, las que se conocieron como "formas clínicas en el viejo

Actualmente, por los cambios sucesivos ocurridos en las sociedades, especialmente durante el pasado siglo, con descensos continuos en la mortalidad y la natalidad, ya es del dominio de todos el progresivo proceso de envejecimiento de las poblaciones, que combina una mayor proporción de adultos mayores con un incremento real de su número y, aún más, de los que llegan a las edades más avanzadas de la vida. ; Estos cambios, son propios de los países más desarrollados. (Omran , 1971) (Omran, 1996)

Aunque sabemos que el desafío del envejecimiento poblacional y la asistencia a las personas mayores es un problema de toda la sociedad y, por tanto, es más social que médico, que requiere de una respuesta global e integrada en cada momento y lugar, es imperioso la repercusión sobre los servicios de salud, la necesidad de formar profesionales de la salud en un contexto demográfico y epidemiológico diferente, situación hasta ahora desconocida por la humanidad. La estrategia actual y futura para enfrentar el reto del cuidado clínico de los adultos mayores no puede estar encargada solamente a la Geriatría, a los geriatras y a otros profesionales y técnicos especializados en esta rama, también se necesita de un personal bien calificado y dedicado a esta disciplina, que oriente y coordine la atención a los ancianos. (Romero Cabrera , 2008)

Los problemas de salud de los adultos mayores requieren del concurso de prácticamente todas las denominadas especialidades clínicas y quirúrgicas, donde tiene un protagonismo especial la Medicina Interna. Hay algunas, aparentemente no relacionadas con los adultos mayores, que cada vez se dedican más a ellos, aún sin contar con una formación básica en el enfoque geriátrico de sus pacientes, enfoque que incluye desde un conocimiento adecuado de la fisiología del envejecimiento, hasta el uso apropiado de medicamentos, por solo mencionar 2 aspectos que se dan por sabidos, pero que con frecuencia no lo son. Entre esas especialidades y servicios, cada vez más presionados con la atención de este grupo poblacional, podemos mencionar, únicamente como botón de muestra, a Ortopedia y 
Traumatología que se enfrenta a la epidemia creciente de fracturas de cadera, a Urología que atiende un número cada vez mayor de enfermos con padecimientos prostáticos, a Cirugía General que asume decisiones complicadas frente a situaciones quirúrgicas nuevas, a Anestesiología que requiere de nuevos conocimientos y habilidades perioperatorias en la asistencia de los ancianos, a Psiquiatría que tiene retos tan cruciales como la depresión y la demencia en la tercera edad y a Cuidados Intensivos que progresivamente ha dado entrada en sus servicios a los enfermos geriátricos críticos. La evaluación geriátrica básica, constituye la piedra angular de la atención clínica a los adultos mayores y consiste en aplicar el método clínico en su dimensión más integral, a los problemas especiales de salud de los adultos mayores. (Espinosa Brito, Romero Cabrera, \& Espinosa Roca, 2009)

\section{Panorama del estado de salud en el adulto mayor}

A medida que la población envejece, aumenta la prevalencia de enfermedades crónicas y discapacitantes. Por lo general, las enfermedades diagnosticadas en los adultos mayores no son curables y, si no se tratan adecuada y oportunamente, tienden a provocar complicaciones y secuelas que dificultan la independencia y la autonomía de las personas.

La salud no puede ser medida solo en términos de la presencia o ausencia de enfermedad, pues se debe valorar también el grado de conservación de la capacidad funcional. La discapacidad acarrea el riesgo de morir, independientemente de la edad, el sexo y de la presencia de otras afecciones. A pesar de que la mayoría de las personas de edad con enfermedades no trasmisibles (ENT) mantienen su capacidad funcional, el grado de discapacidad aumenta con la edad. (Menéndez, y otros, 2005)

Las ECV, la DM, los trastornos cognoscitivos y la depresión tienen efectos independientes en el funcionamiento físico. En general, las ECV, la depresión, la artrosis, la cardiopatía isquémica (CI) y la fractura de la cadera producen el mayor número de casos con discapacidad física en personas de edad avanzada. (Guccione, y otros, 1994)

La hipertensión arterial (HTA) constituye un factor de riesgo, el más importante en el anciano La Organización Panamericana de la Salud estimó que en el transcurso de los próximos 10 años, habrá 
20,7 millones de muertes por enfermedad hipertensiva, en la región de las Américas. Se calcula que la prevalencia de hipertensión en los países latinoamericanos y del Caribe fluctúa entre $8 \%$ y $30 \%$. En el mundo, existe una prevalencia elevada, cada año un número considerable de personas se une al grupo ya existente de hipertensos. (Cruz Betancourt, Martínez García, Lara Delgado, Vargas Ramírez, \& Pérez Pérez, 2015)

La prevalencia de síndrome demencial en América Latina y el Caribe es alta, entre 6,0 y 6,5 por cada 100 adultos de 60 años y más, con un estimado de crecimiento en el número de personas con demencia entre el 2001 y el 2040 del $77 \%$ en los países del cono sur americano (Argentina y Chile) y de 134 a $146 \%$ de incremento en el resto de América Latina, por lo que sobrepasará al de cualquier otra región del mundo. La tasa de incidencia de demencia anual estandarizada para la edad es también elevada, con un estimado de 410938 nuevos casos de demencia por año, la que se asocia a una menor supervivencia en relación con los países de altos ingresos. De 3,4 millones de personas con demencia en América Latina y el Caribe en la actualidad, la cifra se incrementará a 4,1 millones para el 2020 y a 9,1 millones en el 2040, es decir, será similar al de Norteamérica.

Europa Occidental y Norteamérica, presentan las mayores prevalencias de demencia en la población de 60 años o más (7,2 y 6,9 \% respectivamente), seguidas por el Caribe Insular (6,5\%) y Latinoamérica $(6,0 \%)$. La prevalencia de demencia por cada 100 adultos mayores de 60 años, el número estimado de personas con demencia y casos nuevos por año, así como la proporción de incremento en las próximas cuatro décadas y los costos totales que ocasionan las demencias. ( Llibre Rodríguez, \& Gutiérrez Herreral, 2014) 
Diez causas principales de morbilidad -mortalidad en la población de 60 años de edad y mayor, Región de las Américas.

$\checkmark$ Enfermedades isquémicas del corazón.

$\checkmark$ Enfermedades cerebro vasculares

$\checkmark$ Demencia y Alzheimer

$\checkmark$ Enfermedades respiratorias crónicas del tracto inferior

$\checkmark$ Diabetes mellitus

$\checkmark$ Cáncer de tráquea, bronqueo, pulmones

$\checkmark \quad$ Influenza y neumonía

$\checkmark$ Paro cardiaco y enfermedades cardiacas mal definidas

$\checkmark$ Enfermedades Hipertensivas

$\checkmark$ Enfermedades del sistema urinario. (OMS, 2017)

\section{La medicina interna en la asistencia del anciano.}

A pesar de la existencia de la Geriatría como la especialidad que se preocupa de los problemas y enfermedades de los adultos mayores, cómo prevenirlas y manejarlas, y del proceso de envejecer. No sólo del aspecto médico, sino también de aspectos sicológicos y sociales que habitualmente acompañan este proceso, no puede la medicina Interna abstraerse de ese cuidado ya que es el grupo de mayor cantidad de enfermedades presenta con mayor necesidad de la participación del internista en su diagnóstico y solución. (Archila, 2009.) 
Es una estrategia en los servicios de medicina interna que cada vez sean más necesarios médicos que conozcan y atiendan las patologías más prevalentes en el anciano y que aborden el proceso de envejecimiento en su conjunto.

Sin duda el envejecimiento de la población tiene que ver con dos aspectos bien diferenciados. Por un lado, los avances en Medicina Interna que han permitido tratar mejor los procesos agudos ya sea de origen vascular o infeccioso y hacer que muchas enfermedades que hasta ahora eran mortales como el cáncer se transformen en procesos crónicos, permitiendo una calidad de vida adecuada para el individuo. Pero por otro lado y no menos importante, los avances económicos han permitido una importante mejora social y han sido claves en este envejecimiento de la población. Ya no sobreviven los más fuertes sino que todo el mundo tiene prácticamente las mismas opciones. Existe una estrecha relación entre el grado de riqueza de un país y su envejecimiento demográfico. (Pérez Díaz, s.f.) Si bien el tratamiento de los eventos agudos ha contribuido a disminuir la mortalidad por la causa más frecuente de muerte en el mundo occidental que es la enfermedad vascular; en países como EEUU, se considera que la principal causa por la que se ha disminuido la mortalidad causada por las enfermedades coronarias es el mejor control de los factores de riesgo vascular (Ford, y otros, 2007). Por tanto, aún en las personas de edad avanzada sigue siendo fundamental la prevención.

La Medicina Interna es la especialidad médica más completa y más similar en su contenido a la Medicina de Familia, es como si dijéramos la parte hospitalaria de la Medicina Familiar o el equivalente al médico de familia dentro del hospital. Con el paso del tiempo la medicina integral ha dado paso a la medicina basada en parcelas o subespecialidades que con el paso de los años, la adquisición de conocimientos específicos y el desarrollo de nuevas técnicas diagnósticas y terapéuticas se han transformado en especialidades médicas con entidad propia y han dejado al internista la labor integradora de las especialidades y la visión integral de la enfermedad en el cuerpo humano.

Sobre la base de las consideraciones anteriores el servicio de medicina interna pretende brindar una atención clínica personalizada, ágil e íntimamente relacionada con la atención integral de estos 
pacientes pluripatológicos en su mayoría ancianos. El cuidado integral y personalizado acompañado de una formación sanitaria más adecuada del paciente y de su entorno deberían redundar en una atención sanitaria y social coste/efectiva y una mejora en la calidad de vida de estos pacientes. Por otro lado será la forma de afrontar un grave problema sanitario que se nos avecina con el envejecimiento de la población y que ni los internistas, ni los geriatras, ni los gestores podrán solucionar con centros de crónicos. Con este sistema organizativo se evitaría que los ancianos permanecieran durante largas estancias en centros de crónicos. (Pose Reino \& Pena Seijo, 2008)

1. En la práctica, los principales conceptos comunes que mejor definen a esta especialidad son: 1. La visión del enfermo es su conjunto (planteamiento integral de la asistencia) y no de la enfermedad en particular (el internista ve enfermos, no enfermedades).

2. La continuidad asistencial, requisito imprescindible en la calidad de la atención y fundamental en un paciente con enfermedades múltiples crónicas o de edad avanzada, es decir, una gran parte de los pacientes hospitalarios. ${ }^{5}$

3. La coordinación y liderazgo de equipos multidisciplinarios de especialistas y de medicina familiar y comunitaria, así como de interconsultas o programas específicos de salud. El médico internista es interlocutor cuando ha de aunar diversos especialistas porque es la persona, con la mayor capacidad para conocer el ámbito del resto de las especialidades. (Pacheco Mejías, 2017)

Los internistas son los médicos mejor preparados para desenvolverse en los nuevos papeles y salidas profesionales que se presentan en el sistema de salud, al abrir nuevos campos y desarrollar la profesión en áreas como:

1. Unidad de paliativos: en coordinación con el oncólogo, el internista es el indicado para organizar a los pacientes crónicos oncológicos, debido a su formación y experiencia.

2. Asistencia domiciliaria: esta debe ser cubierta por un médico integrador y capaz de tratar todas o casi todas las enfermedades al prescindir de la asistencia de un especialista para cada caso, 
Reflexiones acerca de las enfermedades geriatría y el servicio de medicina interna

además supone un ahorro económico al disminuir el costo de la atención hospitalaria al evitar los reingresos de pacientes con un control domiciliario.

3. Consultas alta resolución: capaces de simplificar procesos y dar altas previas a pacientes que pasan a ser ambulatorios. En la actualidad estas consultas evitan ingresos hospitalarios innecesarios ya que ahorran entre el 30-40 \% de las camas hospitalarias en los países desarrollados.

4. Urgencia y coordinación con urgencias: los internistas son idóneos para las urgencias, ya que su formación es integral, son profesionales polivalentes y pueden atender a cualquier paciente, pues deben articularse en función de parámetros de recambio generacional y contemplarse como una etapa profesional sobre todo para internistas jóvenes.

5. Atención a pacientes ancianos: se convive con la Geriatría, con médicos de atención primaria, trabajadores sociales y se realiza un trabajo integrador.

6. Especialización en áreas muy concretas: los procesos infecciosos, cardiovasculares y oncológicos irán en ascenso, la población demanda resoluciones rápidas en enfermedades agudas pero poco tiempo en el hospital, se busca, por tanto, una medicina más ambulatoria que hospitalaria, para eso hay que mejorar la eficiencia de la hospitalización, como unidades de atención domiciliaria de corta estancia o de alta resolución y en estos campos, los internistas quieren y deben estar presentes.

7. En la atención terciaria: el internista interviene como coordinador entre las diferentes especialidades; tiene a su cargo el diagnóstico y tratamiento de casos críticos o complejos, de enfermedades multiorgánicas, de enfermedades interdisciplinarias e indiferenciadas que rebasan el ámbito de una sola especialidad médica, como las enfermedades del mesénquima, vasculitis o sarcoidosis, las atenciones en emergencia y unidades de cuidados intensivos, áreas que requieren formación integral y amplias destrezas, y aporta con su juicio clínico para uso racional de tecnología

Las enfermedades infecciosas, enfermedades cardiovasculares en pacientes cada vez más complejos (añosos, con más de una enfermedad, plurisintomáticos, oncológicos comórbidos) la Medicina Interna 
en un futuro tendrá un mayor peso en el enfrentamiento en atención con menos estadía sin disminuir la calidad de la misma, también crecerá el papel de médico internista en la mejoría de los circuitos que sigue el paciente (primaria, ambulatoria, hospitalaria, rehabilitación) y en los procesos de deshospitalización o de atención domiciliaria. (Colegio Médico de Ávila , 2014)

En años próximos los servicios de medicina interna asumirán una mayor carga de trabajo en todos los ámbitos de su actividad asistencial. Se centrará de manera esencial en las áreas de diagnóstico, en el tratamiento y manejo de los pacientes más complejos. Y no será solo en las áreas tradicionales sino también en todos los ámbitos intra y extrahospitalarios. El incremento paralelo de la actividad asistencial y de su complejidad clínica es un reto enorme. Enfrentarlo obliga a los internistas a disponer de los conocimientos y habilidades para la atención integral del paciente, disponer de una estructura de atención adecuada y observar exigentes niveles de calidad. (Casariego Lugo , 2016)

Según estudios realizados por (Cinza Sanjurjo, Cabarcos Ortiz de Barrón, Nieto Pol, \& Torre Carballada , 2007) en un Servicio de Medicina Interna arrojo que la prevalencia de patología crónica cardiovascular y pulmonar fue elevada, además de ser las principales causas de mortalidad hospitalaria. De la misma manera según (Llibre Guerral, Milagros, Guerra Hernández, \& Perera Minietll, 2008) plantaron como resultado en su investigación, la hipertensión arterial constituyó la enfermedad de mayor frecuencia y la diabetes mellitus, estuvo presente también con una menor frecuencia el síndrome demencial y la depresión.

En el Hospital General de Perú se realizó un estudio con vista a determinar si existen diferencias en la morbilidad y mortalidad de los adultos mayores de 60 a 79 años hospitalizados, obteniendo como resultado: las patologías de tipo infeccioso fueron las más frecuentes en ambos grupos, existió diferencias en la frecuencia de algunas enfermedades entre los adultos mayores más jóvenes y los más ancianos, la mortalidad en el periodo de estudio y en ambos grupos de adultos mayores no mostró grandes diferencias y sus principales causas también fueron las enfermedades infecciosas. (Rojas, 2010) 
La principal causa de mortalidad y morbilidad en mayores de 65 años fue la bronconeumonía bacteriana, seguida las enfermedades cerebrovasculares y las del corazón. Las principales complicaciones fueron la flebitis y la bronconeumonía hipostática, las cuales predominaron en los fallecidos, estos resultados lo reseño un estudio realizado en el Servicio de Medicina Interna del Hospital "Carlos Manuel de Céspedes. Bayamo Granma. Cuba. (Reyes Tornes \& Card Huerta, 1996)

\section{Conclusión}

Se concluye que la prolongación de la vida, el incremento de la morbilidad y la discapacidad, unido a las políticas de equidad y justicia social, elevan las demandas de atención a los ancianos de servicios de salud con calidad.

\section{Referencias Bibliográficas}

Llibre Rodríguez,, J., \& Gutiérrez Herreral, R. F. (2014). Demencias y enfermedad de Alzheimer en América Latina y el Caribe. Revista Cubana de Salud Pública , 40(3),378-387. Obtenido de https://www.scielosp.org/article/rcsp/2014.v40n3/378-387/

Archila, P. E. (2009.). Los ancianos y la medicina interna Acta Médica Colombiana, 34(3), p. 95.

Benítez Pérez , M. (2017). Envejecimiento poblacional : actualidad y futuro .MediSur ,15(1),8-11. Obtenido de http://scielo.sld.cu/scielo.php?script=sci_arttext\&pid=S1727897X2017000100003\&lng=es\&tlng=es.

Casariego Lugo , E. (2016). El servicio de medicina interna en el siglo XXI. Acta Médica Colombiana; 41(3). Obtenido de www.redalyc.org/pdf/1631/163147636007.pdf

Cinza Sanjurjo, S., Cabarcos Ortiz de Barrón, A., Nieto Pol, E., \& Torre Carballada , A. (2007). Análisis de la mortalidad en ancianos en un Servicio de Medicina Interna. An. Med. Interna (Madrid) 24(2) feb. 
Reflexiones acerca de las enfermedades geriatría y el servicio de medicina interna

Colegio Médico de Ávila . (2014). Médicos y pacientes. Archivo Histórico. Obtenido de htpp://www.icomav.es/pdf/comunicados/MEMORIA\%202014.pdf

Cruz Betancourt, A., Martínez García, E., Lara Delgado, H., Vargas Ramírez, L., \& Pérez Pérez, A. (2015). Morbilidad y mortalidad en adultos mayores, relacionada con la crisis hipertensivas .Correo Científico Médico , 19(4),656-667. Obtenido de http://scielo.sld.cu/scielo.php?script=sci_arttext\&pid=S156043812015000400006\&lng=es\&tlng=es.

Espinosa Brito, A. D., Romero Cabrera, A. J., \& Espinosa Roca, A. A. (2009). La geriatrizacion de los servicios y de los profesionales de la salud. Revista Cubana de Medicina , 48(4),135-138. Obtenido de http://scielo.sld.cu/scielo.php?script=sci_arttext\&pid=S0034$75232009000400001 \& \operatorname{lng}=\mathrm{es} \& \operatorname{tlng}=\mathrm{es}$.

Estudio sobre la protección social de la tercera edad en Ecuador. (2007). Obtenido de https://www.cepal.org/celade/noticias/noticias/1/30081/Doc_Ecuador.pdf

Ford, E., Ajani, U. A., Croft, J. B., Critchley, J. A., Labarthe, D. R., Kottke, T. E., . . Capewell, S. (2007). Explaining the decrease in US deaths from coronary disease, 1980-2000. N Engl J Med ; 356: 2388-98.

González Rodríguez, R., \& Cardentey García, J. (2015). El envejecimiento poblacional : un desafio para los profesionales de la salud en Cuba . Rev Habana de Ciencias Médicas;14(6). Obtenido de http://scielo.sld.cu/scielo.php?script=sci_arttext\&pid=S1729-519X2015000600018

Guccione, A. A., Anderson, J., Anthony, J. M., Zhang, Y., Wilson, P. W., \& a, e. (1994). The effects of specific medical conditions on the functional limitations of elders in the Framingham Study. Am J Public Health. ; 84(3):351 $\square$ 8. 
Llibre Guerral, J. C., Milagros, A., Guerra Hernández, L., \& Perera Minietll, E. (2008). Comportamiento de las enfermedades crónicas no transmisibles en adultos mayores. Rev Cubana Med Gen Integr , 24 (4) oct.-dic..

Louro Bernal, I., Bayarre Vea, H., \& Álvarez Lauzarique, M. E. (2015). Implicaciones familiares y sanitarias del envejecimiento poblacional en la cobertura universal .Rev Cubana Salud Pública ;41 (Suppl 1). Obtenido de http://scielo.sld.cu/scielo.php?script=sci_arttext\&pid=S086434662015000500009

Menéndez, J., Guevara, A., Arcia, N., León Díaz, E. M., Marín, C., \& Alfonso, J. C. (2005). Enfermedades crónicas y limitación funcional en adultos mayores: estudio comparativo en sietes ciudades de América Latina y el Caribe.Revista Panamericana de Salud Pública ,17(56),353-361 . Obtenido de https://www.scielosp.org/article/rpsp/2005.v17n5-6/353-361/

Omran, A. R. (1971). The Epidemiologic Transition. Milkbank Mem Fund, 49:509-38.

Omran, A. R. (1996). The epidemiologic transition in the Americas. Washington DC: Pan American Health Organization.

OMS. (2017). . Salud del adulto mayor. . . Obtenido de https://www.paho.org/salud-en-las-americas2017/?post_t_es=salud-del-adulto-mayor\&lang=es

Pacheco Mejías, A. (2017). Medicina Interna: algunas reflexiones necesarias AMC 21 (6).

Pacheco Mejías, A. (2017). Medicina Interna: algunas reflexiones necesaria. Revista Archivo Médico de Camaguey 21(60,683-686. Obtenido de http://scielo.sld.cu/scielo.php?script=sci_arttext\&pid=S102502552017000600001\&lng=es\&tlng=es.

Pérez Díaz, J. (s.f.). Ventajas internacionales del envejecimiento demográfico. Revista de CIDOB (Fundación Centro de Información y Documentación Internacional de Barcelona. Obtenido de http: www. Ced. Uab.es/jperez. 
Pose Reino, A., \& Pena Seijo, M. (2008). La importancia de la Medicina Interna en la asistencia al anciano, necesidad de creación de unidades específicas. Obtenido de https://dialnet.unirioja.es/descarga/articulo/4242382.pdf

Reyes Tornes, R., \& Card Huerta, C. M. (1996). Morbilidad y mortalidad geriátricas en medicina interna. Rev Cubana Med , 35(2). Obtenido de http://scielo.sld.cu/scielo.php?script=sci_arttext\&pid=S0034$75231996000200005 \& \operatorname{lng}=\mathrm{es} \& n r m=i s o$

Rodríguez Cabrera, A., Collazo Ramos, A., Calero Ricard, J. L., \& Bayarre Vea, H. (2013). Intersectorialidad como vía efectiva para enfrentar el envejecimiento de la población cubana. Rev Cubana Salud Pública ;39(2). Obtenido de http://www.bvs.sld.cu/revistas/spu/vol39_2_13/spu12213.htm

Rodríquez Cabrera , A., Collazo Ramos, M., Álvarez Vázquez, L., Calero Ricardo, J., Castañeda Abascal, I., \& Gálvez González, A. M. (2015). Necesidades de atención en salud percibidad por adultos mayores cubanos. Revista Cubana Salud Pública ;41(3). Obtenido de http://www.scielosp.org/scielo.php?script=sci_arttext\&pid=S0864-34662015000300002

Rojas, D. (2010). Morbilidad y mortalidad del adulto mayor en un servicio de medicina de un hospital general del Perú. Revista Peruana de Epidemiología, 14 (2), 99-107.

Romero Cabrera , A. J. (2008). Perspectivas actuales en la asistencia sanitaria al adulto mayor. Rev Panam Salud Pública. 2008;24(4):288-94. 